Documentation et bibliothèques

DOCUMENTATION BIBLIOTHEQQUES

\title{
Films et vidéos : quelques guides de langue française destinés au grand public
}

\section{Jacques Demers}

Volume 31, numéro 4, octobre-décembre 1985

URI : https://id.erudit.org/iderudit/1052732ar

DOI : https://doi.org/10.7202/1052732ar

Aller au sommaire du numéro

Éditeur(s)

Association pour l'avancement des sciences et des techniques de la documentation (ASTED)

\section{ISSN}

0315-2340 (imprimé)

2291-8949 (numérique)

Découvrir la revue

Citer cet article

Demers, J. (1985). Films et vidéos : quelques guides de langue française destinés au grand public. Documentation et bibliothèques, 31(4), 163-166.

https://doi.org/10.7202/1052732ar

Tous droits réservés (C) Association pour l'avancement des sciences et des techniques de la documentation (ASTED), 1985
Ce document est protégé par la loi sur le droit d'auteur. L'utilisation des services d'Érudit (y compris la reproduction) est assujettie à sa politique d'utilisation que vous pouvez consulter en ligne.

https://apropos.erudit.org/fr/usagers/politique-dutilisation/ 


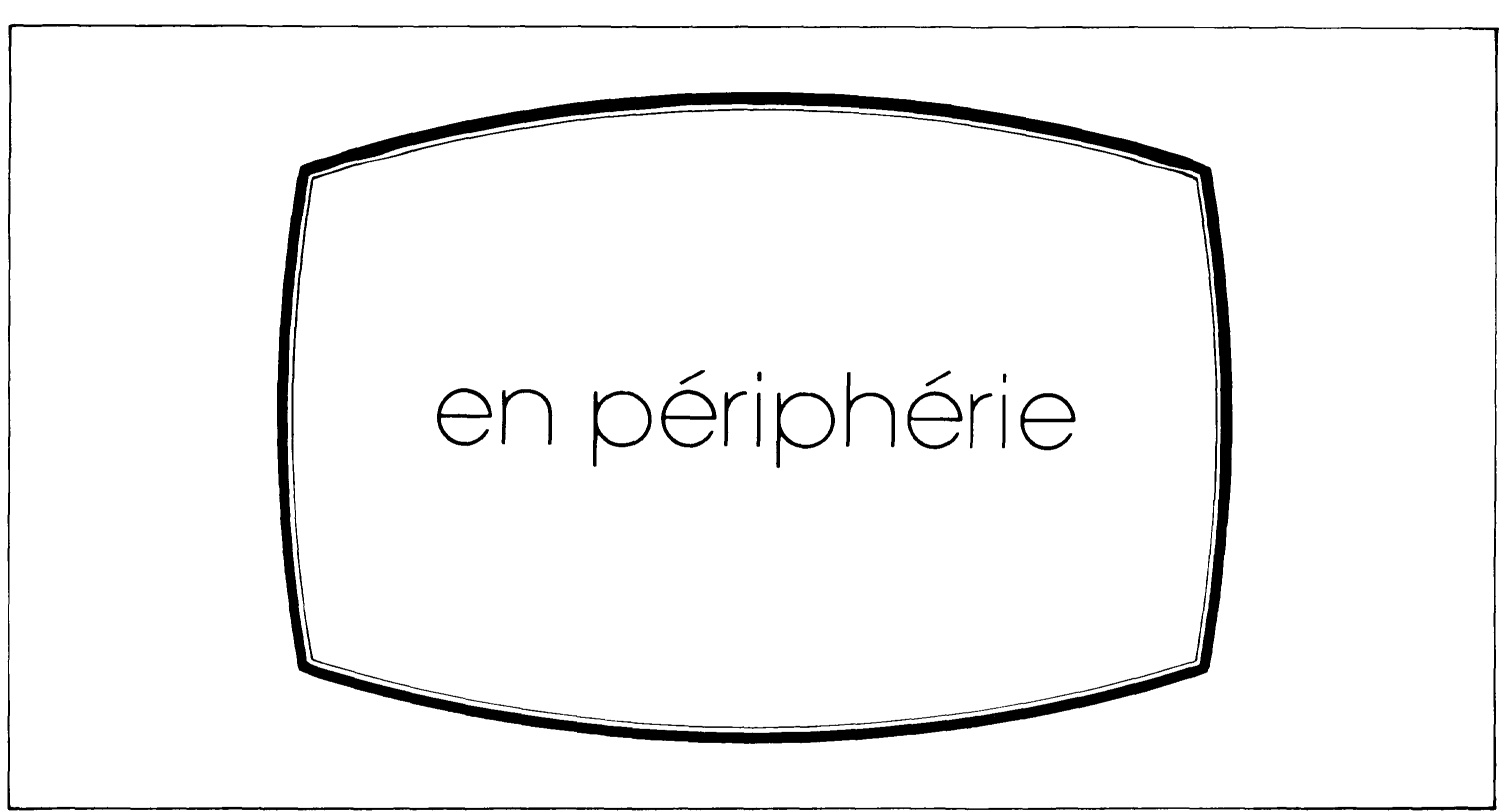

\section{Films et vidéos: quelques guides de langue française destinés au grand public}

Le marché de la vidéo connaît une progression fulgurante depuis le début des années 1980, dans la plupart des pays industrialisés. Le Québec n'échappe pas à ce phénomène. Ainsi, à la fin de 1985,30 à $35 \%$ des foyers québécois possédaient un magnétoscope et on prévoit que ce pourcentage pourrait doubler d'ici 1988.

Cet engouement pour les vidéocassettes a donné naissance chez nous à deux répertoires: Le Guide vidéo $^{1}$ et Le Vidéophile ${ }^{2}$. En France, deux guides ont également vu le jour depuis le début des années 1980: L'Officiel de la vidéo ${ }^{3}$ et le Guide Akaide la vidéo-cassette 4 . Une société française a également publié un répertoire polyvalent, le Cinétéléguide Solar5.

Dans cet article, nous vous proposons une brève analyse de ces cinq instruments. Nous décrivons également le système d'information de l'Office des Communications sociales (OCS) ${ }^{6}$ dans le domaine des films, téléfilms et vidéo. Nous terminerons en tentant de décrire le guide idéal.

\section{Le Guide vidéo}

Ce répertoire, lancé à l'automne 1983, en est à sa troisième édition. II est passé successivement de 2000 à 4500 et maintenant à près de 7000 films recensés. Selon l'introduction, l'ouvrage vise deux objectifs: 1) servir d'outil de référence pour la plupart des films disponibles sur support vidéo; 2) permettre au public de mieux orienter son choix de films.

Les documents répertoriés datent en général de 1960 ou plus, mais le guide présente aussi une sélection limitée de productions antérieures à cette date.

Tous les films sont classés par ordre alphabétique et le titre original est utilisé dans la très grande majorité des cas. La plupart des titres de films sont inscrits sous leur nom français avec renvoi au titre original.

Les notices présentent les informations suivantes: le genre du film, l'année de production, la durée, le nom du réalisateur et des principaux interprètes, la cote d'âge de la Régie du cinéma, un court résumé du contenu et, la plupart du temps, de brefs commentaires. On trouve à la fin de l'ouvrage, un index par genres (33 au total) et des index des acteurs et réalisateurs importants.

La présentation de l'ensemble s'avère très réussie pour un ouvrage vendu à un prix aussi modique.
1. Le Guide vidéo, $3^{\mathrm{e}}$ éd. Québec, Gestion Ciné Vidéo Club, 1985, $722 \mathrm{p}$.

2. Martin Matte, Le vidéophile, Sainte-Foy, Québec, Publications Charles-Huot, 1986, $115 \mathrm{p}$

3. L'Officiel de la vidéo, $5^{e}$ éd., Paris, Télé Ciné Vidéo, 1985, 386 p.

4. Guide Akai de la vidéo-cassette, $3^{e}$ éd., Éditions no 1, 1985, $560 \mathrm{p}$.
5. Cinétéléguide Solar, Paris, Solar, 1984, s.p.

6. L'Office des communications sociales (4005 rue Bellechasse, Montréal, Québec, $\mathrm{H} 1 \mathrm{X}$ 1 J6) publie Films à l'écran depuis 1956. Recueil de Films depuis la même année et Films à la Télé depuis 1959. On lui doit aussi Sélection de films en 16 $\mathrm{mm}$, ouvrage édité en 1978.

7. La Presse, 10 novembre 1985, 78. 
Les notices sont disposées de façon très lisible, le format est pratique et la couverture très attrayante.

Malgré des qualités indéniables, Le Guide vidéo ne contient pas tous les renseignements qu'un amateur exigeant pourrait souhaiter y trouver. II $n$ 'indique pas le nom du distributeur, $n$ 'offre pas de cote d'appréciation, ne précise pas si les versions anglaises et françaises sont disponibles en cassettes. Par ailleurs, il n'est pas fiable à $100 \%$, comme l'a constaté Luc Perrault? 7 . Certains titres disponibles sur support vidéo n'y apparaissent pas et plusieurs films recensés ne sont pas encore édités en vidéocassettes.

Les chiffres suivants donnent une idée de la représentation de certaines catégories de documents: on y trouve 287 films d'animation, 174 documentaires, 176 films musicaux, 133 comédies musicales. II n'y a pas d'entrée pour les films pour enfants, mais la plupart des films d'animation conviennent à un jeune auditoire.

\section{Le Vidéophile}

Dernier-né des guides vidéo, Le Vidéophile se présente sous des allures modestes. Cette première édition recense environ 1500 films disponibles en vidéo. Le choix des titres s'est fait à partir de deux critères: qualité et popularité.

Les films sont classés par ordre alphabétique à l'intérieur de 29 catégories. Les films pornographiques sont carrément exclus et la section drame érotique se limite à 10 titres alors qu'il y a 51 films pour enfants. Le répertoire recense des versions françaises et anglaises. Si un film est disponible dans les deux langues, la notice mentionnera ses titres anglais et français. Dans les autres cas, elle se limite au titre de la version existante.

Outre le ou les titre(s), la notice indique la durée, l'année de production, le(s) pays d'origine, le nom du réalisateur, un court résumé de l'histoire, de brefs commentaires, le nom des principaux interprètes et la classification d'âge de la Régie québécoise du cinéma.

Un index alphabétique des films, classés par genres, précède la section des notices mais l'ouvrage ne comporte aucun autre index.

Le Vidéophile propose des notices dont le contenu et la qualité équivalent en gros à celles du Guide vidéo. Toutefois, ce dernier bénéficie d'une meilleure présentation typographique que Le Vidéophile.

\section{L'Officiel de la vidéo}

II s'agit à notre connaissance du plus ancien guide vidéo édité en langue française puisque la première édition remonte à 1981 et signalait à l'époque 2500 cassettes. Publié par la revue Télé ciné vidéo, L'Officiel de la vidéo paraît en septembre de chaque année.

L'édition 1985 recense 6298 cassettes. Les notices suivent l'ordre alphabétique des titres de la version disponible en France. Toutefois, les cassettes $X$ sont présentées dans une section à part.

\section{Tableau comparatif}

\begin{tabular}{|c|c|c|c|c|c|c|c|c|c|c|c|c|c|c|c|c|c|c|}
\hline \multirow[b]{2}{*}{ 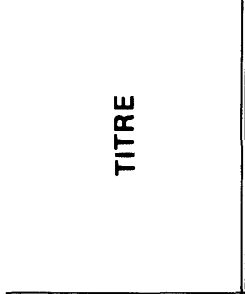 } & \multirow{2}{*}{$\begin{array}{l}\stackrel{n}{u} \\
\stackrel{0}{5} \\
\frac{0}{2}\end{array}$} & \multirow[b]{2}{*}{$\begin{array}{l}z \\
\text { Z } \\
\underline{E} \\
\text { w }\end{array}$} & \multirow{2}{*}{ 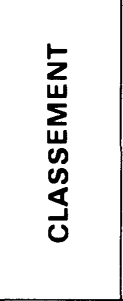 } & \multicolumn{10}{|c|}{ CONTENU DES NOTICES } & \multicolumn{5}{|c|}{ INDEX } \\
\hline & & & & 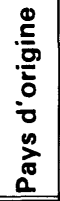 & $\stackrel{8}{\&}$ & 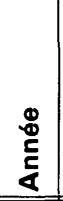 & 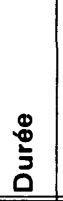 & 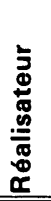 & 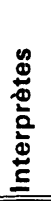 & 离 & 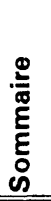 & 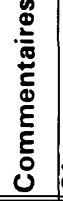 & 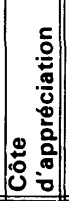 & 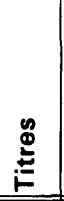 & 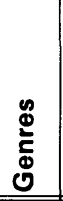 & 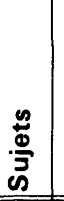 & 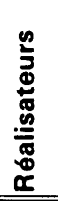 & 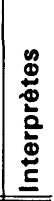 \\
\hline $\begin{array}{c}\text { Le Guide vidéo } \\
13,95 \$\end{array}$ & 7000 & $\begin{array}{c}3 e \\
1985\end{array}$ & titres & $x$ & $x$ & $x$ & $x$ & $x$ & $\mathrm{x}$ & $x$ & $x$ & $\mathrm{x}$ & & & $x$ & & $x$ & $x$ \\
\hline $\begin{array}{c}\text { Le Vidéophile } \\
5,95 \$\end{array}$ & 1500 & \begin{tabular}{|l}
1 ère \\
1986 \\
\end{tabular} & genres & $x$ & $x$ & $x$ & $x$ & $x$ & $x$ & $x$ & $x$ & $x$ & & & $x$ & & & \\
\hline $\begin{array}{c}\text { Guide Akai de la } \\
\text { vidéocassette } \\
16,50 \$\end{array}$ & 1500 & $\begin{array}{c}3 e \\
1985\end{array}$ & genres & $x$ & & $x$ & $x$ & $x$ & $x$ & $x$ & $x$ & $\mathrm{x}$ & $x$ & $x$ & & & $x$ & $x$ \\
\hline $\begin{array}{c}\text { Cinétéléguide } \\
16,50 \$\end{array}$ & 10000 & \begin{tabular}{|l}
1 ère \\
1984
\end{tabular} & titres & $x$ & & $x$ & $x$ & $x$ & $x$ & $x$ & $x$ & & & & & & & $x$ \\
\hline $\begin{array}{l}\text { L'Officiel de la } \\
\text { vidéo } \\
\quad 8,95 \$\end{array}$ & 6298 & $\begin{array}{l}5 e \\
1985\end{array}$ & titres & $x$ & & $x$ & $x$ & $x$ & $x$ & $x$ & $x$ & & $x$ & & $x$ & & & \\
\hline
\end{tabular}


Quoique très succintes, les notices contiennent les renseignements suivants: nom du distributeur, nom du réalisateur, pays d'origine, durée, mention de la couleur, noms des principaux interprètes, résumé du contenu (avec parfois un bref commentaire), le genre sous forme d'un code (26 en tout), l'année de production, une cote d'appréciation sous forme d'étoiles (de une à quatre), et la photo de la jaquette.

On trouve au début de l'ouvrage une section intitulée «Who's who» où l'on présente la liste des films disponibles de 145 réalisateurs importants suivie d'un répertoire des éditeurs français de vidéocassettes avec pour chacun leur adresse, leur numéro de téléphone et leur distributeur. Cet annuel présente à la fin un index où les titres sont regroupés par ordre alphabétique sous 25 catégories. Cet index nous permet de vérifier la répartition des genres; voici quelques exemples: 15 films d'animation, 252 dessins animés, 180 films pour enfants, 153 films érotiques, 43 comédies musicales, 221 films musicaux et 888 films classés.

L'Officiel de la vidéo vise de toute évidence le marché grand public. Ainsi, il est le seul à reproduire la jaquette des documents recensés mais ces photos s'avèrent d'assez mauvaise qualité. Tous les renseignements essentiels y sont mais la concision est de rigueur partout. On se limite à un seul index.

\section{Guide Akai de la vidéocassette}

Le Guide Akai en est à sa troisième édition. Comme ses homologues du disque il se veut sélectif se limitant à présenter environ 1600 des quelque 10000 titres disponibles sur le marché français. Ce choix de 1600 titres d'un bon niveau est présenté par genres. On y retrouve 18 catégories: aventures et action (122), comédies (256), comédies dramatiques (183), comédies musicales et films musicaux (71), dessins animés (12), documentaires (18), drames psychologiques (270), érotiques (33), fantastiques (200), films pour enfants (13), grands classiques (65), grands spectacles (26), guerre (39), karaté (7), policiers (172), politiques (14), western (61), X (50).

Dans chacune de ces catégories, les films sont classés selon l'ordre alphabétique de leur titre français. Pour chacun d'eux on trouve ensuite: une cote d'appréciation (de une à quatre étoiles); l'année de la réalisation; le titre original s'il y a lieu; la nationalité; les noms du réalisateur et des principaux interprètes et, parfois, du compositeur de la musique et du scénariste; un résumé du contenu et d'assez longs commentaires au style très personnel; le distributeur, la durée et la langue de la version, pour les films étrangers.

Trois index complètent l'ouvrage: titres, réalisateurs, principaux interprètes. Toutefois, seul l'index des titres indique la page où se trouve la notice d'un film. Dans les deux autres index on mentionne seulement les titres.

Pour chaque catégorie, l'éditeur propose un surchoix de cinq titres ou plus. L'ouvrage s'avère de consultation agréable grâce à une remarquable disposition des notices

\section{Cinétéléguide Solar}

Cet ouvrage en est à sa première édition. II recense 10000 films présentés selon l'ordre alphabétique de leur titre français ou de leur titre original, lorsque seul ce dernier est connu.

La notice de chaque film contient toujours les renseignements suivants: pays d'origine, année de réalisation, durée lou le métrage ou le nombre de bobines pour les plus anciens), les noms du réalisateur et des principaux interprètes, un résumé du sujet ou de l'action et une indication du genre. On mentionne, lorsqu'il y a lieu, le titre original juste en-dessous du titre français. Une note en petits caractères présente les autres versions tournées sur le même sujet avec dans chaque cas les renseignements essentiels: titre, réalisateur, principaux interprètes, pays d'origine, durée et année. On apprend ainsi que Les misérables de Victor Hugo a inspiré jusqu'à maintenant 13 réalisateurs.

Ce guide ne renferme aucun jugement de valeur. Toutefois, une étoile de sélection identifie quelques chefs-d'œuvre incontestés, au dire de l'éditeur! Une étoile précède des titres comme: Umberto $D$, Citizen Kane, Kagemusha, La ruée vers l'or, Nosferatu le vampire de Murnau, Arsenic et vieilles dentelles. Toutefois, Le cuirassé Potemkine, Le voleur de bicyclette et Cris et chuchottements ne font pas partie de cette sélection. La notion de chef-d'ouvre incontesté apparaît donc pour le moins discutable.

L'ouvrage ne comporte pas véritablement d'index. On trouve, toutefois, à la fin une liste partielle des Oscars et Césars et la filmographie de cent vedettes du cinéma mondial; dans chaque cas, les films sont présentés dans l'ordre alphabétique avec en regard le nom de leur réalisateur et l'année de sortie.

\section{Office des communications sociales}

L'Office des communications sociales (OCS) possède un remarquable système d'information sur les films et vidéos disponibles chez nous. Publiée depuis 1956 (22 livraisons par année), Films à écran recense annuellement un peu plus de 400 films. Les fiches bi-mensuelles sont refondues avec quelques modifications, à la fin de l'année, dans le Recueil des films.

Les notices de ces deux instruments fournissent des renseignements très complets: titre original 
s'il y a lieu, provenance, année, durée, mention de la couleur, genre, réalisateur, principaux interprètes, résumé (assez long), commentaires sur la valeur artistique et morale, cote d'appréciation, distributeur. Les notices de Recueil de films sont classées par ordre alphabétique de titres; les entrées s'établissent au titre original pour les films en langue française et anglaise et au titre de la version anglaise ou française dans les autres cas. Quand le titre original est anglais, un renvoi est établi au titre français. Un index 1956-1980 est disponible et l'OCS en prépare un nouveau pour la période 1956-1985.

L'Office des communications sociales publie aussi depuis 1959, Films à la télé un service destiné à la presse écrite et électronique sur les films à l'horaire des chaînes de télévision qui peuvent être captées au Québec. Films à la télé est extrait d'une base de données informatisée qui comprend quelque 30000 notices. Ces notices contiennent en gros les mêmes renseignements que celles des recueils, mais avec environ $20 \%$ seulement de leur longueur.

Pour faciliter la rédaction des notices de films, I'OCS s'est doté au cours des années d'un fichier de comptes rendus qui comptent maintenant 900000 références. Elle songe actuellement à informatiser ce fichier et à le rendre disponible en accès direct aux cinéphiles intéressés.

L'Office des communications sociales publiait en 1978 Sélection de films en $16 \mathrm{~mm}$, un répertoire regroupant un peu plus de 1500 oeuvres disponibles en français, en anglais ou dans les deux langues. Cet ouvrage utilise les notices de Films à la télé mais avec un système de cotes différent. Un chiffre de 1 à 3 rend compte de l'accessibilité de l'oeuvre et des astérisques (de 1 à 4), de sa valeur artistique. La notice mentionne aussi la classification légale de chaque film et son distributeur. Sélection de films en $16 \mathrm{~mm}$ comprend six parties : 1) notices de films disponibles en français (environ $1325)$; 2) notices de films disponibles en anglais seulement (environ 200); 3) liste des films classés par réalisateurs; 4) films groupés selon certains genres; 5) liste de films classés par thèmes; 6 ) sources littéraires (par ordre alphabétique d'auteurs).

L'OCS ne mettra pas à jour sa Sélection de films en $16 \mathrm{~mm}$ car de moins en moins d'oeuvres sont disponibles sous ce format. Elle songe à publier, à la place, une sélection de films en vidéocassettes qui pourrait recenser de 2000 à 3000 titres.

\section{Le guide idéal}

Quel serait le plan idéal pour un guide de films, téléfilms et vidéos dans le contexte francophone au Canada?
A notre avis, il semble quelque peu utopique d'imaginer qu'un répertoire pourrait à la fois répondre aux attentes de "monsieur tout le monde", de l'amateur averti, du responsable de ciné-club ou du bibliothécaire en charge de la collection vidéos et films dans une bibliothèque.

II nous paraît plus réaliste de songer à deux guides différents. Le premier pourrait ressembler au Guide vidéo. II recenserait, par ordre alphabétique de titres, les documents disponibles sur le marché canadien de la vidéocassette grand public et les films programmés à la télévision.

II pourrait répondre aux deux besoins les plus fréquents des propriétaires de magnétoscopes; faciliter le choix des documents disponibles dans les clubs vidéos; fournir des renseignements sur les films copiés à la télévision.

Ce répertoire grand public devrait fournir une cote d'appréciation pour les produits recensés, soit dans la section des notices soit dans l'index par genres. Cet instrument devrait également offrir des index de réalisateurs et d'interprètes.

Le second guide prendrait la forme d'un instrument sélectif répertoriant de 3000 à 4000 titres, dont environ 1000 documentaires. Les notices des ouvres de fiction pourraient être tirées de la base de données de l'OCS, Films à la télé alors que pour les documentaires on les adapterait des bases FORMAT (Office national du film) et DAVID (Centrale des bibliothèques). Pour chaque document recensé, la notice fournirait les titres des versions disponibles au Canada et le titre original si l'ouvre n'est ni française, ni anglaise.

On indiquerait ensuite le pays d'origine, l'année où le document a été mis en distribution, le nom de la maison de production pour les documentaires, la durée, la mention de la couleur, la classification légale de la Régie québécoise du cinéma, un bref sommaire, quelques commentaires critiques, la mention s'il y a lieu d'oeuvres apparentées, des cotes sur la valeur artistique et l'accessibilité de l'oeuvre et enfin, sous forme abrégée, les noms des distributeurs des copies $35 \mathrm{~mm}, 16 \mathrm{~mm}$ et vidéo.

Ce répertoire serait classé par genres. Cinq index le complèteraient : titres, réalisateurs, interprètes, thèmes et sujets et sources littéraires. $\mathrm{Ce}$ guide aurait-il, toutefois, assez d'usagers pour faire ses frais?

\section{Jacques Demers}

Coordonnateur de l'audiovisuel

Centrale des bibliothèques 